\title{
Susceptibility vessel sign on MRI predicts better clinical outcome in patients with anterior circulation acute stroke treated with stent retriever as first- line strategy
}

\author{
Romain Bourcier, ${ }^{1}$ Wagih Ben Hassen, ${ }^{2}$ Sébastien Soize, ${ }^{3,4}$ Pascal Roux, ${ }^{5}$ \\ Julien Labreuche, ${ }^{6}$ Maeva Kyheng, ${ }^{6}$ Marie Tisserand ${ }_{1}^{7}$ Charlotte Rosso, ${ }^{8}$ \\ Raphael Blanc, ${ }^{5}$ Michel Piotin, ${ }^{5}$ Imad Derraz, ${ }^{9}$ Gregoire Boulouis, ${ }^{2}$ \\ Catherine Oppenheim, ${ }^{2}$ Hubert Desal, ${ }^{1}$ Serge Bracard, ${ }^{10}$ Bertrand Lapergue, ${ }_{1}^{7}$ \\ Olivier Naggara, ${ }^{2,11}$ on behalf of the ASTER and the THRACE investigators
}

- Additional material is published online only. To view please visit the journal online (http://dx.doi.org/10.1136/ neurintsurg-2018-014217).

For numbered affiliations see end of article.

Correspondence to Dr Romain Bourcier, Department of Diagnostic and Interventional Neuroradiology, Guillaume et René Laennec University Hospital, Nantes 44800 S-H, France; romain. bourcier2@gmail.com

$B L$ and $O N$ contributed equally.

Received 25 June 2018 Revised 18 July 2018 Accepted 21 July 2018 Published Online First 28 August 2018

\section{ABSTRACT}

Background Susceptibility vessel sign (SVS) can be a useful MRI biomarker of an occlusion but its relationship with clinical outcomes of acute ischemic stroke (AIS) is yet to be fully elucidated.

Objective To investigate SVS in relation to the clinical outcomes after mechanical thrombectomy using a stent retriever (SR) as first-line approach in patients with AIS. Material and methods We included patients with a first-line SR approach for anterior AIS from the the Contact Aspiration vs Stent Retriever for Successful Revascularization (ASTER) and THRombectomie des Artères CErebrales (THRACE) trials when both baseline imaging of SVS and 90-day modified Rankin Scale (mRS) scores were available. Patients were assigned to two groups based on the presence of an SVS (independent core laboratory), and the overall distributions of the mRS score at 90 days (shift analysis) and clinical independence (mRS score $\leq 2$ ) were compared.

Results 217 patients were included and SVS was diagnosed in $76.0 \%$ of cases $(n=165,95 \% \mathrm{Cl} 70.4 \%$ to $81.7 \%$ ). After adjustment for potential confounders, SVS+ was significantly associated with 90-day mRS improvement (adjusted common OR=2.75; 95\% Cl 1.44 to 5.26) and favorable outcome (adjusted common $\mathrm{OR}=2.76 ; 95 \% \mathrm{Cl} 1.18$ to 6.45 ).

Conclusion Based on results for patients of the ASTER and THRACE trials receiving first-line SR treatment, SVS was associated with lower disability at 3 months. Large prospective studies using MRI-based thrombus evaluation are warranted.

\section{INTRODUCTION}

After demonstration of the superiority of mechanical thrombectomy (MT) over standard medical management alone, the current challenges in the field of acute ischemic stroke (AIS) focus on reducing time to reperfusion, optimizing imaging methods for patient selection, and evaluating the best technical approach. ${ }^{12}$ The Contact Aspiration vs Stent Retriever for Successful Revascularization (ASTER) and the THRombectomie des Artères CErebrales
(THRACE) trials are large multicenter prospective randomized control studies that included patients who were treated with MT for AIS. ${ }^{34}$ In these trials, most of the patients were selected for treatment and inclusion with MRI, allowing thrombus characteristics to be evaluated using the MRI susceptibility vessel sign (SVS). ${ }^{5}$ The SVS can be identified on T2* gradient recalled echo (GRE) sequences and corresponds to a localized hypointense signal at the site of the thrombus, exceeding the diameter of the contralateral artery. ${ }^{56}$ Retrospective monocentric studies have shown that qualitative evaluation of SVS could help predict reperfusion and clinical outcome after MT. ${ }^{7-11}$ The presence of a SVS $(\mathrm{SVS}+)$ is probably related to a larger amount of red blood cells within the thrombus that can favourably interfere with the stent retriever (SR) struts during retrieval. ${ }^{12-15}$ Conversely, its absence (SVS-) has been related to the presence of fibrin-rich thrombi, typically harder to extract with conventional MT using a SR. ${ }^{15-18}$ A SR is the most commonly used technique and, moreover, was the strategy used to show the efficacy of endovascular thrombectomy compared with IV thrombolysis (IVT) alone. Thus, the purpose of our study was to investigate whether SVS + is associated with better clinical and angiographic outcome after MT with SR first-line SR treatment in patients presenting with anterior AIS.

\section{MATERIAL AND METHODS}

The designs of the two trials from which our study population is derived have been already reported. 3419

ASTER examined the question of which firstline strategy for MT (contact aspiration (CA) or SR) leads to higher revascularization rates at the end of the procedure. In line with the recommendations of the American Stroke Association and European Stroke Organization, enrolled patients were given IVT (if eligible) and transferred quickly to the catheter laboratory for urgent $\mathrm{MT}^{2} \mathrm{CA}$ or SR techniques were conducted in accordance with good practice recommendations (minimum of three passes before switching to another strategy; use of a 
proximal occlusion balloon with the SR). The CA approach has been previously reported. ${ }^{20} 21$ The ASTER protocol was registered with ClinicalTrials.gov (Identifier NCT02523261) and the consent forms were approved by an independent institutional review board (Comité de Protection des Personnes Ile de France VI (ID 2015- A00830 -49)).

THRACE aimed to compare IVT alone with IVT plus MT using SR to determine their effects on clinical independence at 3 months in patients with AIS. Patients were included within 4 and 6 hours of symptom onset, respectively, in the THRACE and ASTER trials. The THRACE protocol was approved by an independent institutional review board (Comité de Protection des Personnes III Nord Est Ethics Committee and the research boards of the participating centers). All patients or their legal representatives provided written informed consent. For both trials, practitioners had to show proof of performance of at least five MT procedures before the trial.

\section{Population study}

We included, from the ASTER trial, patients (between October 2015 and October 2016) who received MT with first-line SR treatment and from the THRACE trial, patients (between June 2010 and February 2015) who received IVT plus MT with SR as first line strategy.
Patients with posterior circulation and tandem occlusion were excluded, as were patients for whom baseline SVS information or the 3-month modified Rankin Scale (mRS) scores were not available. The inclusion flowchart is presented in figure 1.

Baseline characteristics, including sex, age, gender, history of hypertension, systolic and diastolic blood pressure, diabetes mellitus, hyperlipidemia, glycemia, smoking habits, initial National Institutes of Health Stroke Scale (NIHSS) score, baseline Alberta Stroke Program Early CT Score (ASPECTS), site of occlusion (either M1/M2 or intracranial carotid artery), IVT use and time between onset and imaging, groin puncture and thrombus contact, were also recorded.

\section{Imaging analysis}

SVS was classified as present (SVS+) or absent (SVS-), according to the definition of Rovira et $a l^{5}$ by independent core laboratories, on the admission T2* MRI sequence. Inter-reader agreement was assessed using un-weighted kappa and 95\% CI in each trial.

\section{Outcomes}

The primary outcome was global disability, assessed by overall distribution of the mRS score at 90 days (shift analysis combining

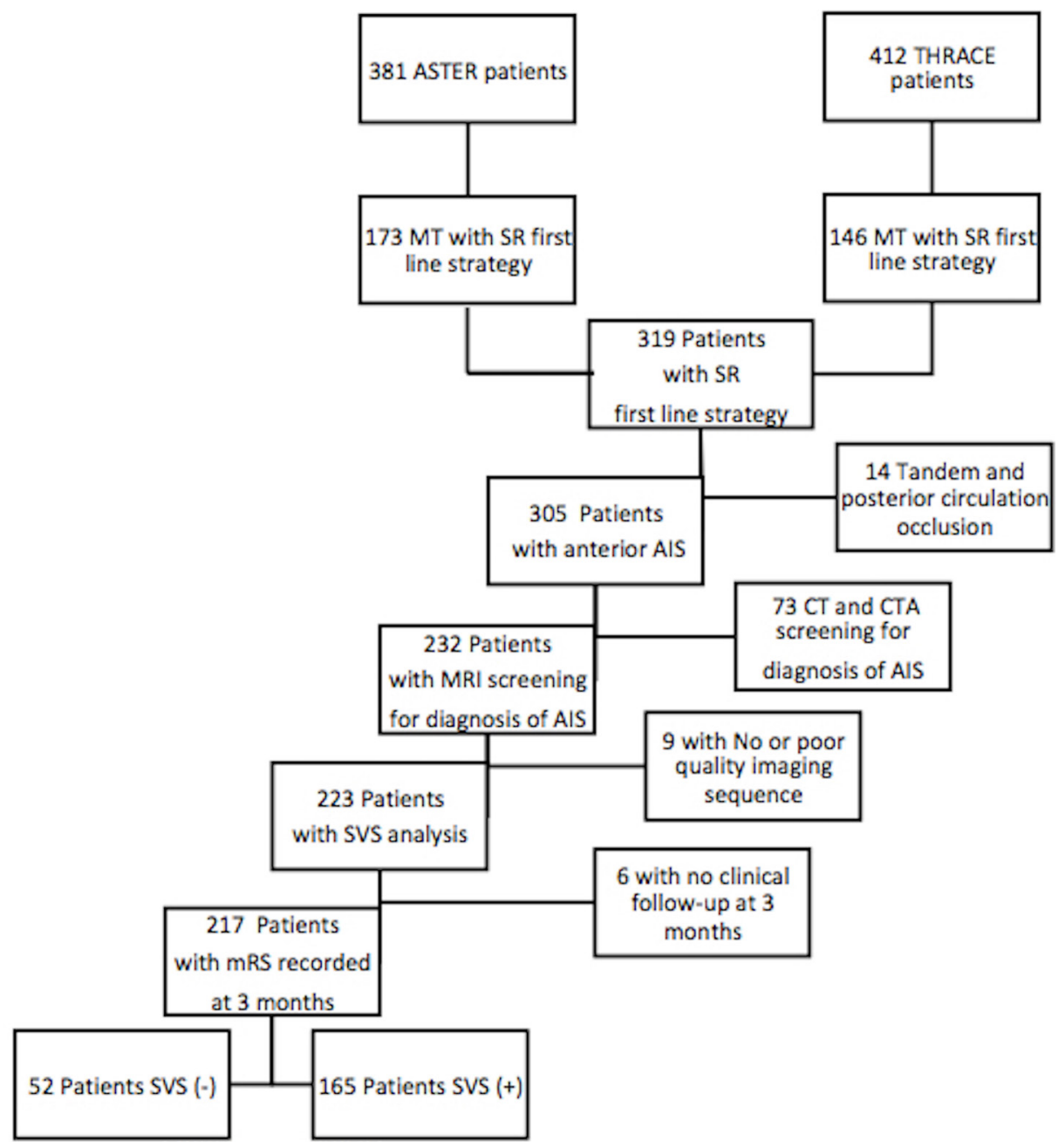

Figure 1 Inclusion flowchart. AIS, anterior ischemic stroke; ASTER, Contact Aspiration versus Stent Retriever for Successful Revascularization; CTA, CT angiography; mRS, modified Rankin Scale; MT, mechanical thrombectomy; SR, stent retriever; SVS, susceptibility vessel sign; THRACE, THRombectomie des Artères CErebrales. 


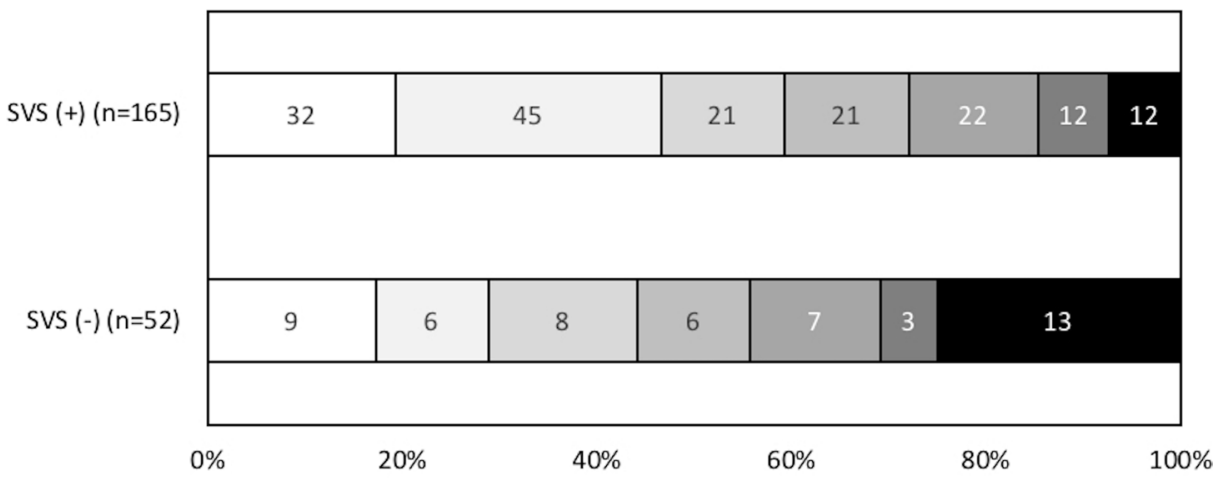

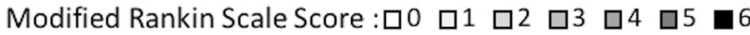

Figure 2 Distribution of modified Rankin Scale score at 90 days according to susceptibility vessel sign (SVS) on MRI.

scores of 5 and 6), and clinical independence as defined by a favorable outcome (90-day mRS score $\leq 2){ }^{22}$

The secondary efficacy outcomes included successful reperfusion rate (defined as a modified Thrombolysis In Cerebral Infarction (mTICI) score of $2 \mathrm{~b}$ or 3 at the end of the MT procedure), ${ }^{23}$ complete reperfusion rate (defined as mTICI 3 at the end of the MT procedure), and the change in NIHSS score at 24 hours.

Safety outcomes included distal emboli in other territories and overall radiological (CT or MRI) intracranial hemorrhage on imaging at $24 \pm 12$ hours.

\section{Statistical analysis}

Categorical variables were expressed as frequencies and percentages. Quantitative variables were expressed as means (SD), or medians (IQR) for non-normal distribution. Normality of distributions was assessed graphically and by the Shapiro-Wilk test. Inter-reader agreement for determination of SVS was assessed using the simple Cohen's kappa coefficient. Baseline characteristics were compared between patients with and without SVS using logistic regression adjusted for the study (ASTER or THRACE) using SVS status as an independent variable. Comparisons of angiographic and clinical outcomes between patients with and without SVS were performed after adjustment for the study using logistic regression models for binary outcomes, an ordinal logistic regression model for overall 90 -day mRs score (shift analysis after combining scores 5 and 6), and a covariance analysis for 24-hour NIHSS adjusted on baseline values. We assessed the between-study heterogeneity for each outcome by including the corresponding interaction term in previous models. Finally, between-group comparisons of outcomes were further adjusted for prespecified confounding factors (study, age, and admission NIHSS) and baseline differences (at $\mathrm{P}<0.10$ in bivariate analyses). Using patients without SVS as reference, effect sizes for binary outcomes were calculated as odds ratios (ORs), for overall distribution of mRS scores as a common OR for one point in improvement, and for quantitative outcomes as mean between-group differences.

All statistical tests were two-sided and $\mathrm{P}<0.05$ was considered as significant. Data were analyzed using the SAS software package, release 9.4 (SAS Institute, Cary, North Carolina, USA).

\section{RESULTS}

Of the 793 patients randomized in the ASTER and THRACE trials, 319 patients were treated with a SR and 217 of them were eligible for inclusion in this study (figure 1). Reasons for exclusion were posterior or tandem occlusion $(n=14)$, absence or poor, non-diagnostic quality of admission brain MRI $(\mathrm{n}=82)$, and missing 90-day follow-up $(n=6)$. Main baseline characteristics and outcomes of the study population are available in the online supplementary table.

SVS + was observed in $76.0 \%$ of cases $(n=165,95 \%$ CI $70.4 \%$ to $81.7 \%$ ) with a significant difference between the two trials $(67.2 \%(\mathrm{n}=84)$ in ASTER vs $88.0 \%(\mathrm{n}=81)$ in THRACE, $\mathrm{P}<0.001)$. Inter-reader agreements for determination of SVS were 0.83 (95\% CI 0.80 to 0.86 ) and 0.88 (95\% CI 0.81 to 0.94), respectively, in the THRACE and ASTER trials.

At the end of the MT procedure, a successful reperfusion rate was observed in $80.6 \%(\mathrm{n}=175,95 \%$ CI $75.3 \%$ to $85.9 \%)$, favourable outcome in $55.8 \% \quad(\mathrm{n}=121 ; 95 \% \mathrm{CI} 49.1 \%$ to $62.4 \%$ ), distal emboli in other territories in $3.2 \%$, only present in the SVS + group ( $\mathrm{n}=7 ; 95 \% \mathrm{CI} 1.0 \%$ to $6.0 \%$ ), and any intracranial hemorrhage in $40.6 \%(\mathrm{n}=88 ; 95 \%$ CI $34.0 \%$ to $47.1 \%)$.

As shown in table 1, patients with SVS were more often men, non-diabetic, and had an M1/M2 occlusion, and a longer onsetto-MRI time than patients without SVS, although none of the differences reached significance.

shows the distributions of mRS scores at 90 days according to the presence or absence of SVS. In study-adjusted analysis, we found a significant difference in distribution of 90-day mRS scores, with lower scores in the SVS + group than in the SVS - group, with a common OR for one-point improvement of 2.26 (95\% CI 1.27 to 4.03). Compared with SVS- patients, those from the SVS+ group had a significantly higher favorable outcome rate $(\mathrm{OR}=2.03 ; 95 \% \mathrm{CI} 1.06$ to 2.91$)$ and a greater 24-hour NIHSS score decrease (mean between-group difference, $-3.2 ; 95 \% \mathrm{CI}-6.1$ to -0.3 ). There was no difference in reperfusion rates (complete or successful) or incidence of intracranial hemorrhage within 24 hours (table 1). No significant heterogeneity in association with SVS for each study outcome was found (all $\mathrm{P}>0.20$ ).

After adjustment for potential confounding factors, SVS remained significantly associated with an improvement in 90-day mRS score (adjusted common $\mathrm{OR}=2.75 ; 95 \% \mathrm{CI} 1.44$ to 5.26) and favorable outcome (adjusted common $\mathrm{OR}=2.76$; $95 \%$ CI 1.18 to 6.45 ). However, the association of SVS with 24 hours decrease in NIHSS was no longer significant (table 2).

\section{DISCUSSION}

Using aggregate data from two randomized controlled trials dedicated to endovascular treatment of AIS with first-line SR, we demonstrated that SVS+, assessed on pretreatment brain MRI, is a strong predictor of favorable clinical outcome. 
Table 1 Baseline characteristics according to susceptibility vessel sign (SVS) on MRI

\begin{tabular}{|c|c|c|c|}
\hline Characteristics & $\begin{array}{l}\text { SVS- } \\
(n=52)\end{array}$ & $\begin{array}{l}\text { SVS+ } \\
(n=165)\end{array}$ & $P$ valuest \\
\hline \multicolumn{4}{|l|}{ Demographics } \\
\hline Age, years, mean (SD) & $65.7(14.1)$ & $64.5(15.4)$ & 0.98 \\
\hline Men & $21 / 52(40.4)$ & $91 / 165(55.2)$ & 0.095 \\
\hline \multicolumn{4}{|l|}{ Medical history } \\
\hline Hypertension & $33 / 52(63.5)$ & 79/161 (49.1) & 0.17 \\
\hline Diabetes & $13 / 52(26.5)$ & $17 / 163(10.4)$ & 0.066 \\
\hline Hypercholesterolemia & 19/49 (38.8) & $60 / 155(38.7)$ & 0.89 \\
\hline Current smoking & $8 / 45(17.8)$ & $33 / 143(23.1)$ & 0.49 \\
\hline Coronary artery disease & 9/51 (17.6) & $21 / 161(13.0)$ & 0.78 \\
\hline \multicolumn{4}{|l|}{ Current stroke event } \\
\hline $\begin{array}{l}\text { Systolic blood pressure, } \\
\mathrm{mmHg} \text {, mean (SD)‡ }\end{array}$ & $142.1(26.1)$ & $142.1(22.9)$ & 0.85 \\
\hline $\begin{array}{l}\text { Diastolic blood pressure, } \\
\text { mmHg, mean (SD) }\end{array}$ & $81.0(17.4)$ & $81.0(15.1)$ & 0.86 \\
\hline Glycemia, median (IQR)§ & 6.6 (5.6 to 7.7$)$ & 6.7 (5.9 to 8.2$)$ & 0.071 \\
\hline NIHSS score, mean (SD)ף & $16.2(6.0)$ & $16.7(5.6)$ & 0.91 \\
\hline ASPECTS, median (IQR) ** & 7 (5 to 8) & 7 (5 to 8) & 0.66 \\
\hline Site of occlusion & & & 0.082 \\
\hline $\mathrm{M} 1 / \mathrm{M} 2$ & $40 / 52(76.9)$ & $144 / 165(87.3)$ & \\
\hline ICA & $12 / 52(23.1)$ & $21 / 165(12.7)$ & \\
\hline Intravenous rt-PA & $35 / 52(67.3)$ & $141 / 165(85.5)$ & 0.15 \\
\hline Etiology & & & 0.38 \\
\hline Atherothrombotic & $8 / 51(15.7)$ & $17 / 161(10.6)$ & \\
\hline Cardioembolic & $17 / 51(33.3)$ & 72/161 (44.7) & \\
\hline Other or unknown causes & $26 / 51(51.0)$ & $72 / 161(44.7)$ & \\
\hline Favorable collaterality & 10/42 (23.8) & 26/139 (18.7) & 0.56 \\
\hline \multicolumn{4}{|l|}{$\begin{array}{l}\text { Intervals times, min, median } \\
\text { (IQR) }\end{array}$} \\
\hline $\begin{array}{l}\text { Onset to groin puncture } \\
\text { timet† }\end{array}$ & 220 (165 to 296$)$ & 215 (180 to 255$)$ & 0.57 \\
\hline Onset to imaging $\ddagger$ & 102 (72 to 135$)$ & $115(88.5$ to 141$)$ & 0.089 \\
\hline Onset to clotł‡ & 252 (200 to 325$)$ & 248 (210 to 285 ) & 0.57 \\
\hline
\end{tabular}

Values expressed as the no/total no (\%) unless otherwise indicated.

$P$ values were computed using logistic regression model adjusted for study (except for Intravenous rt-PA).

tP values calculated after log- transformation of data.

‡Two missing values (one in SVS+).

§Seven missing values (four in SVS+).

qOne missing value in SVS-

${ }^{* *}$ Four missing values (three in SVS+).

††Three missing values (two in SVS+).

$\ddagger \ddagger$ Five missing values (four in SVS+).

ASPECTS, Alberta Stroke Programme Early CT Score; ICA, internal carotid artery; IQR, IQR range; $M 1, M 2$, first or second segment of the middle cerebral artery; NIHSS, National Institutes of Health Stroke Scale; rt-PA, recombinant tissue plasminogen activator; SVS, susceptibility vessel sign.

Our findings are in line with a previous smaller retrospective monocentric study, in which SVS+ appeared to be a radiologic biomarker predictive of favorable clinical outcome at 3 months after $\mathrm{MT} .{ }^{10}$ However, a very large CI precluded any generalization of the results $(\mathrm{OR}=8.7 ; 95 \% \mathrm{CI} 1.1$ to 69.4 ; $\mathrm{P}$ value $0.04)$. Our results are in apparent contradiction with those of others, ${ }^{724}$ which did not establish SVS+ as a treatment effect imaging biomarker. In the overall population of the ASTER trial, SR use has recently shown its superiority for recanalization and clinical outcome compared with CA when SVS is present. However, conversely, in this group of patients treated with SR and CA, SVS was not found to be a factor related to clinical outcome. This might be due, in part, to the merging of SR- and CA-treated patients but also because the sample size in the SR group was small $(59.0 \%$ in SVS + vs $43.1 \%$ in SVS-; adjusted risk ratio (RR), 1.27 ; $95 \%$ CI 0.97 to 1.66$).{ }^{16}$ Because SR is the most commonly used technique and, moreover, the strategy used to show the efficacy of endovascular thrombectomy compared with IVT alone, we thought it important to highlight the prognostic significance of SVS. Hence, we collected a large, controlled population of patients treated with SR, and showed that, when treated with SR, the presence of SVS is a prognostic factor related to good outcome. Conversely, the absence of SVS in patients treated with SR is related to unfavorable outcome.

Despite the better clinical outcome in the SVS + group, mTICI scores did not differ between groups. SVS is observed when red blood cell-dominant clots are present, whereas a lack of SVS is indicative of clots with a higher fibrin content or an underlying atherosclerotic plaque. ${ }^{9142526}$ In the latter case, one may argue that an SVS - patient might have experienced delayed shortterm reocclusion, despite a final mTICI $2 \mathrm{~b} / 3 .{ }^{27-29}$ Even if we were unable to assess the vessel patency at 24 or 48 hours after MT, we found a trend towards a higher NIHSS score at 24 hours in the SVS - group, which might reflect such a reocclusion. Furthermore, the observed impact of an SVS - occlusion on clinical outcome may be explained by a higher number of passes required to reach a TICI $2 \mathrm{~b} / 3$ reperfusion. ${ }^{30} 31$ The thrombus composition and its mechanical properties could explain a more difficult MT procedure, requiring more numerous passes. Indeed, a fibrin-rich thrombus can be difficult to engage in the SR, as it adheres more strongly to the vessel wall, ${ }^{15} 17$ and SVS - is thought to be related to fibrin-rich thrombi. However, we were unable to analyse the number of passes in this study.

Potential limitations should be considered in interpreting the results of this report.

First, differences in entry criteria and patient characteristics among the trials are a source of potential bias. Second, no thrombus histology data were available to further characterize the relationships between SVS, outcomes, and thrombus composition. ${ }^{32-34}$

Third, a favorable collateral circulation has been associated with better reperfusion and subsequent more favorable clinical outcomes. $^{35}$ We did not adjust our analysis for these collaterals since we included M1/M2 but also internal carotid artery occlusions for which presence of collaterals with DSA was not recorded. Fourth, we analysed SVS only as a binary variable (presence/absence), ${ }^{5}$ whereas others distinguished different subtypes of SVS (namely 'two-layered SVS') or carried out a quantitative estimation based on the overestimation ratio of SVS. ${ }^{36-38}$

Finally, although SVS+ identification is reliable and reproducible, with excellent interobserver agreement, SVS evaluation was performed on different MRI machines owing to the multicentric design of both studies. This is a significant limitation, as it was previously reported in an in vitro study that the prevalence of the SVS+ varies significantly among MRI machines. ${ }^{26}$

\section{CONCLUSION}

Basing our study on the ASTER and THRACE trial populations treated with SR as a first-line strategy, we found a higher rate of favourable clinical outcome at 3 months in SVS+ patients. Thrombus MRI examination before MT might be 
Table 2 Comparison of main angiographic and clinical outcomes according to susceptibility vessel sign (SVS) on MRI

\begin{tabular}{|c|c|c|c|c|c|c|c|}
\hline \multirow[b]{2}{*}{ Outcomes } & \multirow{2}{*}{$\begin{array}{l}\text { SVS- } \\
(n=52)\end{array}$} & \multirow{2}{*}{$\begin{array}{l}\text { SVS+ } \\
(n=165)\end{array}$} & \multirow[b]{2}{*}{ Effect size } & \multicolumn{2}{|l|}{ Study-adjusted } & \multicolumn{2}{|l|}{ Fully adjusted } \\
\hline & & & & Values $(95 \% \mathrm{Cl})$ & P Values & Values $(95 \% \mathrm{Cl})^{*}$ & P Values* \\
\hline \multicolumn{8}{|l|}{ Angiographic outcomes } \\
\hline $\mathrm{mTICl} 3$ & $20 / 52(38.5)$ & $53 / 165(32.1)$ & OR & 0.95 (0.48 to 1.86$)$ & 0.88 & 0.96 (0.45 to 2.06$)$ & 0.92 \\
\hline $\mathrm{mTICl} 2 \mathrm{~b} / 3$ & $42 / 52(80.8)$ & 133/165 (80.6) & OR & 1.34 (0.59 to 3.17 ) & 0.47 & 1.31 (0.51 to 3.38 ) & 0.58 \\
\hline $\begin{array}{l}\text { Favorable outcome at } \\
3 \text { months }\end{array}$ & $23 / 52(44.2)$ & 98/165 (59.4) & OR & 2.03 (1.06 to 3.91$)$ & 0.034 & 2.76 (1.18 to 6.45$)$ & 0.019 \\
\hline $\begin{array}{l}\text { Change in NIHSS at } 24 \\
\text { hours§ }\end{array}$ & $-3.1(-5.6$ to -0.6$)$ q & $-6.3(-7.7$ to -5.0$)$ ๆ & Mean difference** & $-3.20(-6.04$ to -0.35$)$ & 0.028 & $-2.59(-5.66$ to 0.48$)$ & 0.098 \\
\hline $\begin{array}{l}\text { Intracranial hemorrhage at } \\
24 \text { hours }\end{array}$ & $21 / 51(41.2)$ & $67 / 164(40.9)$ & OR & 0.99 (0.51 to 1.92 ) & 0.98 & 0.98 (0.47 to 2.05$)$ & 0.96 \\
\hline
\end{tabular}

Values expressed as the no/total no (\%), unless otherwise stated. OR and mean difference were calculated using patients with SVS (-) as the reference group.

*Adjusted for prespecified factors (study, age, admission NIHSS) and baseline between-group difference at $\mathrm{P}<0.10$ in bivariate analysis (gender, diabetes, IVT, glycemia, site of occlusion, onset to MRI time).

tMedian (IQR).

$\ddagger$ Common OR of improvement of 1 point in 90-day mRS score.

$\S 13$ missing values (five in SVS-).

ๆMean change $(95 \% \mathrm{Cl})$ adjusted for study and baseline values.

**Adjusted mean between-group difference.

IVT, IV thrombolysis; mRS, modified Rankin Scale; mTICI, modifiedThrombolysis in Cerebral Infarction score; NIHSS, National Institutes of Health Stroke Scale;

SVS, susceptibility vessel sign.

useful for choosing a first-line strategy in acute ischemic stroke following large vessel occlusions. Large prospective studies using MRI-based thrombus evaluation are warranted.

\section{Author affiliations}

'Department of Diagnostic and Interventional Neuroradiology, Guillaume et René Laennec University Hospital, Nantes, France

${ }^{2}$ Department of Neuroradiology, Université Paris<Descartes. INSERM U894, Sainte Anne Hospital, Paris, France

${ }^{3}$ Department of Diagnostic and Interventional Neuroradiology, University Hospital of Reims, Reims, France

${ }^{4}$ INSERM UMR<S 1237 Physiopathology and Imaging of Neurological Disorders, Université Caen Normandie, Caen, France

${ }^{5}$ Department of Diagnostic and Interventional Neuroradiology, Rothschild Foundation, Paris, France

${ }^{6}$ Department of Biostatistics, University Lille, CHU Lille, EA 2694-Santé Publique: Epidémiologie et Qualité des Soins, Lille, France

${ }^{7}$ Department of Stroke Center and Diagnostic and Interventional Neuroradiology, University of Versailles and Saint Quentin en Yvelines, Foch Hospital, Suresnes, France ${ }^{8}$ Urgences cérébro-vasculaires, Sorbonne Université, UPMC Univ Paris 06, Hôpital Pitié<Salpêtrière, Paris, France

${ }^{9}$ Department of Diagnostic and Interventional Neuroradiology, Hôpital Gui de Chauliac, Montpellier, France

${ }^{10}$ Department of Diagnostic and Interventional Neuroradiology, University Hospital of Nancy, Nancy, France

${ }^{11}$ Pediatric Radiology Department, Necker Enfants Mala des, Paris, France

Acknowledgements We thank Malek Ben Maacha, MSc, clinical research associate (Department of Diagnostic and Interventional Neuroradiology, Rothschild Foundation, France) for serving as the main clinical research associate for the trial and we thank Mary Osborne<Pellegrin for her help in editing the final draft of the article. They did not receive specific compensation for their work related to this study.

Collaborators The THRACE trial investigators are Alain Bonafé (Department of Neuroradiology, Gui de Chauliac Hospital, Montpellier, France), Xavier Leclerc (Department of Radiology, University Hospital of Lille, Lille, France), Nelly Agrinier (Department of Clinical Epidemiology INSERM CIC-EC 1433, University of Lorraine and University Hospital of Nancy, Nancy, France), Serge Bakchine (Department of Neurology, University Hospital of Reims, Reims, France), Flore Baronnet (Stroke Unit, Pitié-Salpétrière Hospital Group and Paris 6 Université Pierre et Marie Curie, Paris, France), Marine Beaumont (Department of INSERM CIC-IT, University of Lorraine and University Hospital of Nancy, Nancy, France), Yannick Bejot (Department of
Neurology, University Hospital of Dijon, Dijon, France), Jerome Berge (Department of Interventional and Diagnostic Neuroradiology, University Hospital of Bordeaux, Bordeaux, France), Marc Bintner (Department of Neuroradiology Sud-Reunion Hospital Group, Saint Pierre, France), Romain Bourcier (Department of Interventional and Diagnostic Neuroradiology, University Hospital of Nantes, Nantes, France), Tae Hee Cho (Department of Neurology, University Hospital of Lyon, Lyon, France), Frédéric Clarençon (Department of Interventional Neuroradiology Pitié-Salpétrère Hospital Group and Paris 6 Universityé Pierre et Marie Curie, Paris, France), Julien Cogez (Department of Neurology, University Hospital of Caen, Caen, France), Charlotte Cordonnier (Department of Neurology, University Hospital of Lille, Lille, France), Christian Denier (Department of Neurology, University Hospital of Bicètre, Le Kremlin-Bicètre, France), Anne Laure Derelle (Department of Diagnostic and Interventional Neuroradiology, University Hospital of Nancy, Nancy, France), Olivier Detante (Department of Neurology, University Hospital of Grenoble, Grenoble, France), Anthony Faivre (Department of Neurology, Hopital d'Instruction des Armées, Sainte Anne, Toulon, France), Anne Ferrier, (Department of Neurology, University Hospital Gabriel-Montpied, Clermont-Ferrand, France), Laetitia Gimenez (Department of Neurology, University Hospital of Limoges, Limoges, France), Sophie Godard (Department of Neurology, University Hospital of Angers, Angers, France), Benoit Guillon (Department of Neurology, University Hospital of Nantes, Nantes, France), Emmanuel Houdart (Department of Neuroradiology, University Hospital Lariboisière, Paris, France), Bertrand Lapergue (Department of Neurology, Foch Hospital, Suresnes, France), Mariano Musacchio (Department of Neuroradiology, Pasteur Hospital, Colmar, France), Olivier Naggara (Department of Neuroradiology, Sainte-Anne Hospital and Paris-Descartes University, INSERM U894, Paris, France), Jean Philippe Neau (Department of Neurology, University Hospital of Poitiers, Poitiers, France), Michael Obadia (Department of Neurology, Rothschild Ophthalmological Foundation, Paris, France), Anne Pasco-Papon (Department of Radiology, University Hospital of Angers, Angers, France), Michel Piotin (Department of Interventional Neuroradiology [MP] Rothschild Ophthalmological Foundation, Paris, France), Laurent Pierot (Department of Neuroradiology, University Hospital of Reims, Reims, France), Helene Raoult (Department of Neuroradiology, University Hospital of Rennes, Rennes, France), Sébastien Richard (Department of Neurology University Hospital of Nancy, Nancy, France), Frederic Ricolfi (Department of Neuroradiology, University Hospital of Dijon, Dijon, France), Thomas Ronziere (Department of Neurology, University Hospital of Rennes, Rennes, France), Guillaume Saliou (Department of Neuroradiology, University Hospital of Bicètre, Le Kremlin-Bicètre, France), Igor Sibon (Department of Neurology, University Hospital of Bordeaux, Bordeaux, France), Sebastien Soize (Department of Neuroradiology, University Hospital of Reims, Reims, France), Jacques Sedat (Department of Radiology, University Hospital of Nice, Nice, France), Christian 
Stapf (Department of Neurology, University Hospital Lariboisière, Paris, France), Laurent Suissa (Department of Neurology, University Hospital of Nice, Nice, France), Marie Tisserand (Department of Neuroradiology, Sainte-Anne Hospital and ParisDescartes University, INSERM U894, Paris, France), Francis Turjman (Department of Interventional Neuroradiology, University Hospital of Lyon, Lyon, France), and Stephane Velasco (Departments of Radiology, University Hospital of Poitiers, Poitiers, France). The ASTER trial Investigators are: Piotin Michel, Blance Raphael, Redjem Hocine, Mazighi Mickael, Fahed Robert, Desilles Jean Philippe, Lapergue Bertrand, Rodesch Georges, Consoli Arturo, Di Maria Federico, Turiman Francis, Gory Benjamin, Labeyrie Paul Emile, Riva Roberto, Mounayer Charbel, Saleme Suzana, Costalat Vincent, Bonafé Alain, Eker Omer, Gascou Grégory, Dargazanli Cyril, Bracard Serge, Tonnelet Romain, Derelle Anne Laure, Anxionnat René, Desal Hubert, Bourcier Romain, Daumas-Duport Benjamin, Berge Jérome, Barreau Xavier, Margnat Grégoire, Labreuche Julien.

Contributors $\mathrm{RB}$ conceived the idea for the study and wrote the manuscript. ON, $B L$, and WBH wrote the manuscript. SS, PR, MT, CR, RB, MP, ID, GB, CO, HD and SB collected data and made a critical review of the manuscript. JL and MK performed the statistical analysis.

Funding The ASTER trial research was sponsored by Fondation Ophtalmologique Adolphe de Rothschild. An unrestricted research grant was provided by Penumbra, Alameda, California. The THRACE trial was funded by the French Ministry for Health as part of its 2009 STIC programme for the support of costly innovations (grant number 2009 A00753-54).

\section{Competing interests None declared.}

Patient consent Obtained.

Ethics approval The ASTER protocol was registered with ClinicalTrials.gov (Identifier NCT02523261) and the consent form was approved by the Comite de Protection des Personnes lle de France VI (ID 2015- A00830 -49). The THRACE trial aimed to compare intravenous thrombectomy (IVT) alone with IVT plus mechanical thrombectomy using SR to determine their effect on clinical independence at 3 months in patients with acute ischemic stroke. Patients were included within respectively 4 and 6 hours of symptom onset in the THRACE and ASTER trial. The THRACE protocol was approved by the Comite de Protection des Personnes III Nord Est Ethics Committee and the research boards of the participating centers. All patients or their legal representatives provided written informed consent.

Provenance and peer review Not commissioned; externally peer reviewed.

Data sharing statement Data are available upon request from the corresponding author.

\section{REFERENCES}

1 Goyal M, Menon BK, van Zwam WH, et al. Endovascular thrombectomy after large-vessel ischaemic stroke: a meta-analysis of individual patient data from five randomised trials. Lancet 2016;387:1723-31.

2 Powers WJ, Derdeyn CP, Biller J, et al. 2015 American Heart Association/American Stroke Association focused update of the 2013 guidelines for the early management of patients with acute ischemic stroke regarding endovascular treatment: a guideline for healthcare professionals from the American Heart Association/American Stroke Association. Stroke 2015:46:3020-35.

3 Bracard S, Ducrocq X, Mas JL, et al. Mechanical thrombectomy after intravenous alteplase versus alteplase alone after stroke (THRACE): a randomised controlled trial. Lancet Neurol 2016;15:1138-47.

4 Lapergue B, Blanc R, Gory B, et al. Effect of endovascular contact aspiration vs stent retriever on revascularization in patients with acute ischemic stroke and large vessel occlusion: the ASTER randomized clinical trial. JAMA 2017:318:443.

5 Rovira A, Orellana P, Alvarez-Sabín J, et al. Hyperacute ischemic stroke: middle cerebral artery susceptibility sign at echo-planar gradient-echo MR imaging. Radiology 2004;232:466-73.

6 Flacke $\mathrm{S}$, Urbach $\mathrm{H}$, Keller $\mathrm{E}$, et al. Middle cerebral artery (MCA) susceptibility sign at susceptibility-based perfusion MR imaging: clinical importance and comparison with hyperdense MCA sign at CT. Radiology 2000;215:476-82.

7 Soize S, Batista AL, Rodriguez Regent C, et al. Susceptibility vessel sign on T2* magnetic resonance imaging and recanalization results of mechanical thrombectomy with stent retrievers: a multicentre cohort study. Eur J Neurol 2015;22:967-72.

8 Kimura K, Sakamoto Y, Iguchi Y, et al. Clinical and MRI scale to predict very poor outcome in tissue plasminogen activator patients. Eur Neurol 2011:65:291-5.

9 Kim SK, Yoon W, Heo TW, et al. Negative susceptibility vessel sign and underlying intracranial atherosclerotic stenosis in acute middle cerebral artery occlusion. AJNR Am J Neuroradiol 2015;36:1266-71
10 Bourcier R, Volpi S, Guyomarch B, et al. Susceptibility vessel sign on MRI predicts favorable clinical outcome in patients with anterior circulation acute stroke treated with mechanical thrombectomy. AJNR Am J Neuroradio/ 2015;36:2346-53.

11 Aoki J, Kimura K, Shibazaki K, et al. Location of the susceptibility vessel sign on t2* -weighted MRI and early recanalization within 1 hour after tissue plasminogen activator administration. Cerebrovasc Dis Extra 2013:3:111-20.

12 Hashimoto T, Hayakawa M, Funatsu N, et al. Histopathologic analysis of retrieved thrombi associated with successful reperfusion after acute stroke thrombectomy. Stroke 2016;47:3035-7.

13 Bourcier R, Brecheteau N, Costalat $\mathrm{V}$, et al. MRI quantitative T2* mapping on thrombus to predict recanalization after endovascular treatment for acute anterior ischemic stroke. J Neuroradiol 2017;44:241-6.

14 Liebeskind DS, Sanossian N, Yong WH, et al. CT and MRI early vessel signs reflect clot composition in acute stroke. Stroke 2011;42:1237-43

15 Machi P, Jourdan F, Ambard D, et al. Experimental evaluation of stent retrievers' mechanical properties and effectiveness. J Neurointerv Surg 2017;9:257-63.

16 Bourcier R, Mazighi M, Labreuche J, et al. Susceptibility vessel sign in the aster trial: higher recanalization rate and more favourable clinical outcome after first line stent retriever compared to contact aspiration. J Stroke 2018;20:268-76.

17 Fennell VS, Setlur Nagesh SV, Meess KM, et al. What to do about fibrin rich 'tough clots'? Comparing the Solitaire stent retriever with a novel geometric clot extractor in an in vitro stroke model. J Neurointerv Surg 2018:neurintsurg-2017-013507.

18 Gunning GM, McArdle K, Mirza M, et al. Clot friction variation with fibrin content; implications for resistance to thrombectomy. J Neurointerv Surg 2018;10:34-8.

19 Lapergue B, Labreuche J, Blanc R, et al. First-line use of contact aspiration for thrombectomy versus a stent retriever for recanalization in acute cerebral infarction: the randomized ASTER study protocol. Int J Stroke 2018;13:87-95.

20 Turk AS, Frei D, Fiorella D, et al. ADAPT FAST study: a direct aspiration first pass technique for acute stroke thrombectomy. J Neurointerv Surg 2014;6:260-4.

21 Kowoll A, Weber A, Mpotsaris A, et al. Direct aspiration first pass technique for the treatment of acute ischemic stroke: initial experience at a European stroke center. J Neurointerv Surg 2016;8:230-4.

22 Savitz SI, Lew R, Bluhmki E, et al. Shift analysis versus dichotomization of the modified Rankin scale outcome scores in the NINDS and ECASS-II trials. Stroke 2007:38:3205-12.

23 Tomsick T, Broderick J, Carrozella J, et al. Revascularization results in the interventiona management of stroke II trial. AJNR Am J Neuroradiol 2008:29:582-7.

24 Naggara O, Raymond J, Domingo Ayllon M, et al. T2* "susceptibility vessel sign" demonstrates clot location and length in acute ischemic stroke. PLoS One 2013;8:e76727.

25 Baik SH, Kwak HS, Hwang SB, et al. Three-dimensional black blood contrast enhanced magnetic resonance imaging in patients with acute ischemic stroke and negative susceptibility vessel sign. Eur J Radiol 2018;102:188-94.

26 Bourcier R, Détraz L, Serfaty JM, et al. MRI interscanner agreement of the association between the susceptibility vessel sign and histologic composition of thrombi. J Neuroimaging 2017:27:577-82.

27 Yoo AJ, Andersson T. Thrombectomy in acute ischemic stroke: challenges to procedural success. J Stroke 2017:19:121-30.

28 Kang DH, Kim YW, Hwang YH, et al. Instant reocclusion following mechanical thrombectomy of in situ thromboocclusion and the role of low-dose intra-arterial tirofiban. Cerebrovasc Dis 2014;37:350-5.

$29 \mathrm{Kim} \mathrm{BM}$. Causes and solutions of endovascular treatment failure. J Stroke 2017:19:131-42.

30 Angermaier A, Michel P, Khaw AV, et al. Intravenous thrombolysis and passes of thrombectomy as predictors for endovascular revascularization in ischemic stroke. J Stroke Cerebrovasc Dis 2016;25:2488-95.

31 Linfante I, Starosciak AK, Walker GR, et al. Predictors of poor outcome despite recanalization: a multiple regression analysis of the NASA registry. J Neurointerv Surg 2016:8:224-9.

32 Boeckh-Behrens T, Schubert M, Förschler A, et al. The impact of histological clot composition in embolic stroke. Clin Neuroradio/ 2016:26:189-97.

33 Marder VJ, Chute DJ, Starkman S, et al. Analysis of thrombi retrieved from cerebra arteries of patients with acute ischemic stroke. Stroke 2006;37:2086-93.

34 Brinjikji W, Duffy S, Burrows A, et al. Correlation of imaging and histopathology of thrombi in acute ischemic stroke with etiology and outcome: a systematic review. J Neurointerv Surg 2017:9:529-34.

35 Bang OY, Saver JL, Kim SJ, et al. Collateral flow predicts response to endovascular therapy for acute ischemic stroke. Stroke 2011:42:693-9.

36 Kang DW, Jeong HG, Kim DY, et al. Prediction of stroke subtype and recanalization using susceptibility vessel sign on susceptibility-weighted magnetic resonance imaging. Stroke 2017;48:1554-9.

37 Zhang R, Zhou Y, Liu C, et al. Overestimation of susceptibility vessel sign: a predictive marker of stroke cause. Stroke 2017:48:1993-6.

38 Yamamoto N, Satomi J, Tada Y, et al. Two-layered susceptibility vessel sign on 3-tesla T2* ${ }^{*}$-weighted imaging is a predictive biomarker of stroke subtype. Stroke 2015;46:269-71. 